\title{
A CLOSED GRAPH THEOREM FOR ORDER BOUNDED OPERATORS
}

\author{
JAN HARM VAN DER WALT \\ Department of Mathematics and Applied Mathematics, University of Pretoria, Pretoria, \\ 0002, South Africa. \\ E-Mail janharm.vanderwalt@up.ac.za
}

\begin{abstract}
The closed graph theorem is one of the cornerstones of linear functional analysis in Fréchet spaces, and the extension of this result to more general topological vector spaces is a difficult problem comprising a great deal of technical difficulty. However, the theory of convergence vector spaces provides a natural framework for closed graph theorems. In this paper we use techniques from convergence vector space theory to prove a version of the closed graph theorem for order bounded operators on Archimedean vector lattices. This illustrates the usefulness of convergence spaces in dealing with problems in vector lattice theory, problems that may fail to be amenable to the usual Hausdorff-Kuratowski-Bourbaki concept of topology.
\end{abstract}

Mathematics Subject Classification (2010): Primary 46A40, 47B65; Secondary 46A19.

Key words: Vector lattice, convergence vector space, order bounded operator.

1. Introduction. Analysis on vector lattices comprises two related issues, namely, the structural theory of vector lattices dealing with order and algebraic properties of vector lattices, and topological type phenomena such as convergence of sequences and the accompanying notions of continuity of linear operators. This paper concerns aspects of the latter of these issues. While notions of convergence and continuity are clearly fundamental topological phenomena, such notions that typically arise in the study of vector lattices can, in general, not be described solely in terms of the classical Hausdorff-Kuratowski-Bourbaki concept of topology. Indeed, neither order convergence, nor relatively uniform convergence of sequences can, in general, be induced by a topology on a vector lattice $X$ through the relation

(1.1) $\left(x_{n}\right)$ converges to $x \in X \Leftrightarrow\left(\begin{array}{ll}\forall & V \text { a neighbourhood of } x: \\ \exists & N \in \mathbb{N}: \\ \forall & n \in \mathbb{N}, n \geq N: \\ & x_{n} \in V\end{array}\right)$,

see for instance $[10$, Section 16] and $[3,11,16]$. However, as is shown in $[3,11,16]$ and in this paper, both order convergence and relatively uniform convergence of sequences are induced by convergence structures [8]. Thus the theory of convergence spaces, and convergence vector spaces in particular, provides an appropriate context for, as well as powerful tools with which to study topological phenomena on vector lattices. In the present paper we give an example of how methods from 
the theory of convergence vector spaces may be applied to problems in the theory of vector lattices. In particular, we prove a closed graph theorem for order bounded operators on Archimedean vector lattices.

As mentioned in the abstract, the closed graph theorem is one of the fundamental theorems of analysis in topological vector spaces. The first version, proved in the setting of Fréchet spaces, is due to Banach [4]. Subsequently, generalisations of this theorem to more general classes of topological vector spaces were given by Pták [12], Słowikowski [15], Raikov [13] and de Wilde [9].

In the context of convergence vector spaces, the results of Pták and de Wilde find a natural setting. In particular, the proof of Pták's theorem is difficult to understand from a technical point of view, since it essentially makes use of duality arguments, which are not generally available in locally convex spaces. On the other hand, the continuous convergence structure provides a beautiful duality theory for locally convex spaces [8], thus giving dramatic insight into and clarification of Pták's results, see for instance [5] and [7]. Furthermore, de Wilde's webbed spaces fit nicely into the convergence vector space setting, see $[6,8]$. With these remarks in mind, it is therefore clear that convergence vector spaces provide an excellent setting for the study of closed graph theorems.

The paper is organized as follows. In Section 2 we show that the Mackey modification of the order convergence structure induces relatively uniform convergence of sequences. In Section 3 it is shown how this convergence structure fits into the framework of the closed graph theorem for convergence vector spaces $[8$, Theorem 6.2.6]. A closed graph theorem for order bounded operators is then proved. For details on convergence spaces and vector lattices we refer the reader to [8] and [10], respectively.

Let us now fix some notation. Throughout, $X$ will denote an Archimedean vector lattice. For $x \in X,[x]$ denotes the principle ultrafilter generated by $x$, that is, $[x]=\{F \subseteq X: x \in F\}$. If $\left(x_{n}\right)$ is a sequence in $X$, then $\left\langle x_{n}\right\rangle=\left[\left\{\left\{x_{n}: n \geq\right.\right.\right.$ $k\}: k \in \mathbb{N}\}$ ] is the Fréchet filter associated with $\left(x_{n}\right)$. The neighbourhood filter at $0 \in \mathbb{R}$ is denoted by $\mathcal{N}$.

2. Relatively uniform convergence. In this section we study relatively uniform convergence on an Archimedean vector lattice $X$. We will show that, on any such $X$, there exists a vector space convergence structure that induces relatively uniform convergence. In this regard, we recall [10] the following basic facts. A sequence $\left(x_{n}\right)$ converges relatively uniformly to $x \in X$ if

$$
\begin{array}{ll}
\exists & \mu \in X^{+}: \\
\forall & \epsilon>0: \\
\exists & N_{\epsilon} \in \mathbb{N}: \\
& \left|x-x_{n}\right| \leq \epsilon \mu, n \geq N_{\epsilon} .
\end{array}
$$

More generally, a sequence $\left(x_{n}\right)$ is a relatively uniformly Cauchy sequence whenever

$$
\begin{array}{ll}
\exists & \mu \in X^{+}: \\
\forall & \epsilon>0: \\
\exists & N_{\epsilon} \in \mathbb{N}: \\
& \left|x_{m}-x_{n}\right| \leq \epsilon \mu, m, n \geq N_{\epsilon} .
\end{array}
$$


The vector lattice $X$ is called relatively uniformly complete if every relatively uniformly Cauchy sequence converges relatively uniformly in $X$.

A sequence $\left(x_{n}\right)$ order converges to $x \in X$ whenever

$$
\exists\left(\mu_{n}\right) \subset X:
$$

1) $0 \leq \mu_{n+1} \leq \mu_{n}, n \in \mathbb{N}$

2) $\inf \left\{\mu_{n}: n \in \mathbb{N}\right\}=0$

3) $\left|x-x_{n}\right| \leq \mu_{n}, n \in \mathbb{N}$.

As is the case for relatively uniform convergence, there is in general no topology on $X$ that induces order convergence of sequences. However, the order convergence structure $\lambda_{o}$, introduced in [3], induces order convergence of sequences, see $[3,16]$. The order convergence structure is defined as follows: For each $x \in X, \lambda_{o}(x)$ is the collection of filters $\mathcal{F}$ on $X$ that satisfy

$$
\begin{aligned}
& \exists \quad\left(\lambda_{n}\right),\left(\mu_{n}\right) \subset X: \\
& \text { 1) } \lambda_{n} \leq \lambda_{n+1} \leq \mu_{n+1} \leq \mu_{n}, n \in \mathbb{N} \\
& \text { 2) } \sup \left\{\lambda_{n}: n \in \mathbb{N}\right\}=x=\inf \left\{\mu_{n}: n \in \mathbb{N}\right\} \\
& \text { 3) }\left[\left\{\left[\lambda_{n}, \mu_{n}\right]: n \in \mathbb{N}\right\}\right] \subseteq \mathcal{F} .
\end{aligned}
$$

The order convergence structure is a first countable and Haudorff vector space convergence structure. We denote $X$ equipped with the order convergence structure by $o(X)$.

We now show that relatively uniform convergence is induced by a vector space convergence structure. In particular, the Mackey modification $\lambda_{r}$ of the order convergence structure induces relatively uniform convergence.

Proposition 2.1. A set $U \subset X$ is bounded with respect to the order convergence structure if and only if there exists $\mu \geq 0$ so that $U \subseteq[-\mu, \mu]$.

Proof. Suppose that $U \subset o(X)$ is bounded. Then the filter $\mathcal{U}=\mathcal{N} U$ converges to 0 in $o(X)$ so that

$$
\begin{aligned}
& \exists\left(\mu_{n}\right) \subset X: \\
& \text { 1) } \quad 0 \leq \mu_{n+1} \leq \mu_{n}, n \in \mathbb{N} \\
& \text { 2) } \quad \inf \left\{\mu_{n}: n \in \mathbb{N}\right\}=0 \\
& \text { 3) } \quad \mathcal{G}=\left[\left\{\left[-\mu_{n}, \mu_{n}\right]: n \in \mathbb{N}\right\}\right] \subseteq \mathcal{U} .
\end{aligned}
$$

The filter $\mathcal{U}$ has basis $\left\{U_{m}: m \in \mathbb{N}\right\}$, where

$$
U_{m}=\left\{\epsilon x: x \in U,|\epsilon|<\frac{1}{m}\right\}, m \in \mathbb{N} .
$$

Since $\mathcal{G} \subseteq \mathcal{U}$, it therefore follows that

$$
\begin{aligned}
\forall & n \in \mathbb{N}: \\
\exists & m_{n} \in \mathbb{N}: \\
& U_{m_{n}} \subseteq\left[-\mu_{n}, \mu_{n}\right] .
\end{aligned}
$$


In particular, $-\mu_{1} \leq \frac{1}{2 m_{1}} x \leq \mu_{1}$ for all $x \in U$ so that $U=\subseteq[-\mu, \mu]$ where $\mu=2 m_{1} \mu_{1}$.

Conversely, if $U \subseteq[-\mu, \mu]$ for some $\mu \in X^{+}$, then $-\mu_{n} \leq \epsilon x \leq \mu_{n}$ for all $n \in \mathbb{N}$, $|\epsilon|<\frac{1}{n}$ and $x \in U$, where $\mu_{n}=\frac{1}{n} \mu$. Therefore $\left[\left\{\left[-\mu_{n}, \mu_{n}\right]: n \in \mathbb{N}\right\}\right] \subseteq \mathcal{N} U$ and, since $\left(\mu_{n}\right)$ decreases to 0 , it follows that $\mathcal{N} U$ converges to 0 in $o(X)$. Hence $U$ is bounded in $o(X)$.

The following is now immediate.

Corollary 2.2. A filter $\mathcal{F}$ on $X$ converges to 0 with respect to the Mackey modification $\lambda_{r}$ of the order convergence structure if and only if

$$
\begin{array}{ll}
\exists & \mu \in X^{+}: \\
& {\left[\left\{\left[-\frac{1}{n} \mu, \frac{1}{n} \mu\right]: n \in \mathbb{N}\right\}\right] \subseteq \mathcal{F} .}
\end{array}
$$

We denote the Archimedean vector lattice $X$ equipped with the convergence structure $\lambda_{r}$ by $r(X)$. Next we show that the Mackey modification of the order convergence structure induces relatively uniform convergence of sequences.

Theorem 2.3. A sequence $\left(x_{n}\right)$ in $X$ converges relatively uniformly to $x \in X$ if and only if $\left(x_{n}\right)$ converges to $x$ in $r(X)$.

Proof. Suppose that $\left(x_{n}\right)$ converges relatively uniformly to $x \in X$. Let $\mu$ be the positive element of $X$ associated with $\left(x_{n}\right)$ through (2.1). Then

$$
\begin{array}{ll}
\forall & k \in \mathbb{N}: \\
\exists & N_{k} \in \mathbb{N}: \\
& \left\{x-x_{n}: n \geq N_{k}\right\} \subseteq\left[-\frac{1}{k} \mu, \frac{1}{k} \mu\right]
\end{array}
$$

so that $\left\langle x_{n}\right\rangle-[x] \supseteq\left[\left\{\left[-\frac{1}{n} \mu, \frac{1}{n} \mu\right]: n \in \mathbb{N}\right\}\right]$. Therefore the sequence $\left(x_{n}\right)$ converges to $x$ in $r(X)$.

Conversely, suppose that the sequence $\left(x_{n}\right)$ converges to $x$ in $r(X)$. Then there exists $\mu \in X^{+}$so that $\left[\left\{\left[-\frac{1}{n} \mu, \frac{1}{n} \mu\right]: n \in \mathbb{N}\right\}\right] \subseteq\left\langle x_{n}\right\rangle-[x]$. Thus

$$
\begin{array}{ll}
\forall & k \in \mathbb{N}: \\
\exists & N_{k} \in \mathbb{N}: \\
& \left\{x_{n}-x: n \geq N_{k}\right\} \subset\left[-\frac{1}{k} \mu, \frac{1}{k} \mu\right] .
\end{array}
$$

Hence, for each $k \in \mathbb{N},\left|x-x_{n}\right|<\frac{1}{k} \mu$ for all $n \geq N_{k}$ so that $\left(x_{n}\right)$ converges relatively uniformly to $x$.

Completeness of the convergence vector space $r(X)$ is characterized in terms of relatively uniform completeness of $X$, as we now show.

Proposition 2.4. The convergence vector space $r(X)$ is complete if and only if $X$ is relatively uniformly complete. 
Proof. Since the Mackey modification of a convergence vector space is first countable [8, Section 3.7], it follows by [8, Proposition 3.6.5] that $r(X)$ is complete if and only if it is sequentially complete. Therefore we need only show that the Cauchy sequences in $r(X)$ are precisely the relatively uniformly Cauchy sequences. In this regard, let $\left(x_{n}\right)$ be a relatively uniformly Cauchy sequence in $\mathrm{X}$, and $\mu$ the positive element of $X$ associated with $\left(x_{n}\right)$ through (2.2). Then

$$
\begin{array}{ll}
\forall & k \in \mathbb{N}: \\
\exists & N_{k} \in \mathbb{N}: \\
& \left\{x_{m}-x_{n}: m, n \geq N_{k}\right\} \subset\left[-\frac{1}{k} \mu, \frac{1}{k} \mu\right] .
\end{array}
$$

But the filter $\left\langle x_{n}\right\rangle-\left\langle x_{n}\right\rangle$ is based on the collection of sets

$$
\left\{\left\{x_{m}-x_{n}: m, n \geq N\right\}: N \in \mathbb{N}\right\}
$$

so that $\mathcal{G}=\left[\left\{\left[-\frac{1}{n} \mu, \frac{1}{n} \mu\right]: n \in \mathbb{N}\right\}\right] \subseteq\left\langle x_{n}\right\rangle-\left\langle x_{n}\right\rangle$. Since $\mathcal{G}$ converges to 0 in $r(X)$, it follows that $\left\langle x_{n}\right\rangle$ is a Cauchy sequence in $r(X)$.

Conversely, let $\left(x_{n}\right)$ be a Cauchy sequence in $r(X)$, and $\mu$ the positive element of $X$ associated with the filter $\left\langle x_{n}\right\rangle-\left\langle x_{n}\right\rangle$ through Corollary 2.2. Since

$$
\left[\left\{\left[-\frac{1}{n} \mu, \frac{1}{n} \mu\right]: n \in \mathbb{N}\right\}\right] \subseteq\left\langle x_{n}\right\rangle-\left\langle x_{n}\right\rangle,
$$

it follows that

$$
\begin{array}{ll}
\forall & k \in \mathbb{N}: \\
\exists & N_{k} \in \mathbb{N}: \\
& \left\{x_{m}-x_{n}: m, n \geq N_{k}\right\} \subset\left[-\frac{1}{k} \mu, \frac{1}{k} \mu\right] .
\end{array}
$$

Therefore

$$
\begin{array}{ll}
\forall & \epsilon>0: \\
\exists & N_{\epsilon} \in \mathbb{N}: \\
& \left|x_{m}-x_{n}\right| \leq \epsilon \mu, m, n \geq N_{\epsilon}
\end{array}
$$

so that $\left(x_{n}\right)$ is a relatively uniformly Cauchy sequence.

3. Closed graph theorems. In this section we apply the convergence vector spaces introduced in Section 2 to obtain closed graph theorems for operators defined on suitable relatively uniformly complete Archimedean vector lattices. In particular, we will prove such a result for order bounded operators. The main tool in his regard is the following, see [8, Theorem 6.2.6].

TheOrem 3.1. Let $E$ be an inductive limit of Fréchet spaces and $F$ a convergence vector space admitting a finer vector space convergence structure which is ultracomplete. Then any closed linear mapping $T: E \rightarrow F$ is continuous.

The larger part of this section is devoted to showing how the convergence vector space $r(X)$ fits into the setting of the Closed Graph Theorem 3.1. This consists of two problems. Firstly, we have to show that the convergence structure on $r(X)$ 
is coarser than some ultracomplete vector space convergence structure on $X$. This is not true in general, and we characterize those vector lattices $X$ that do admit an ultracomplete convergence structure that is finer than $\lambda_{r}$. On the other hand, $r(X)$ has to be expressed as the convergence inductive limit of a family of Fréchet spaces.

Let us first consider the latter of these two issues. In this regard, we consider, for each strictly positive $u \in X$, the principle ideal $I(u)$ generated in $X$ by $u$. On each of these principle ideals, we consider the following association of filters with points in $I(u)$.

Definition 3.2. A filter $\mathcal{F}$ on $I(u)$ belongs to $\lambda_{u}(0)$ whenever

$$
\left[\left\{\left[-\frac{1}{n} u, \frac{1}{n} u\right]: n \in \mathbb{N}\right\}\right] \subseteq \mathcal{F}
$$

For $x \neq 0$, a filter $\mathcal{F}$ belongs to $\lambda_{u}(x)$ if $\mathcal{F}-[x] \in \lambda_{u}(0)$.

Note that we can introduce a norm on $I(u)$ by setting

$$
\|x\|_{u}=\inf \left\{\alpha \in \mathbb{R}^{+}:|x| \leq \alpha u\right\}
$$

for every $x \in I(u)$. An easy computation shows that (3.1) does in fact define a norm on $I(u)$. Moreover, the filter $\left[\left\{\left[-\frac{1}{n} u, \frac{1}{n} u\right]: n \in \mathbb{N}\right\}\right]$ is the neighbourhood filter at 0 with respect to the topology induced on $I(u)$ by $(3.1)$. Therefore $\lambda_{u}$ is the natural convergence structure induced by the norm topology on $I(u)$, see $[8$, Example 1.1.2 (i)]. Thus we have the following:

Corollary 3.3. If $X$ is relatively uniformly complete, then $I(u)$ is a Fréchet space for every strictly positive $u \in X$. In particular, if $X$ is relatively uniformly complete and has a strong order unit e, then $r(X)$ is a Fréchet space.

Note that Corollary 3.3 can be strengthened in the following way: If $X$ is relatively uniformly complete, then $I(u)$ is a Banach lattice for every strictly positive $u \in X$. Furthermore, if $X$ is relatively uniformly complete and has a strong order unit $e$, then $r(X)$ is a Banach lattice. This is a well known result, see for instance [14, Proposition 7.2 \& Cororally]. However, for the purpose of this paper, the result as it is stated is sufficient.

We now show that $r(X)$ is the convergence inductive limit of the Fréchet spaces $I\left(u_{i}\right)$, with $u_{i} \in X$ strictly positive. In this regard, we note that the family of ideals $\left\{I\left(u_{i}\right): u_{i}>0\right\}$ is upward directed with respect inclusion and

$$
X=\bigcup_{u_{i}>0} I\left(u_{i}\right)
$$

Furthermore, with respect to the convergence structures $\lambda_{u_{i}}$ on each of the ideals $I\left(u_{i}\right)$, the inclusion mappings

$$
e_{i j}: I\left(u_{i}\right) \ni x \mapsto x \in I\left(u_{j}\right), 0<u_{i}<u_{j}
$$


and

$$
e_{i}: I\left(u_{i}\right) \ni x \mapsto x \in r(X), u_{i}>0
$$

are continuous. Indeed, this follows immediately from the identities

$$
e_{i j}([0, x])=\left[e_{i j}(0), e_{i j}(x)\right]=\left[0, e_{i j}(x)\right], e_{i}([0, x])=\left[e_{i}(0), e_{i}(x)\right]=\left[0, e_{i}(x)\right],
$$

$x \in I\left(u_{i}\right)$, and the definitions of the convergence structures on the spaces $I\left(u_{i}\right)$ and $r(X)$, respectively. Moreover, for all $0<u_{i}<u_{j}<u_{k}$, the diagrams
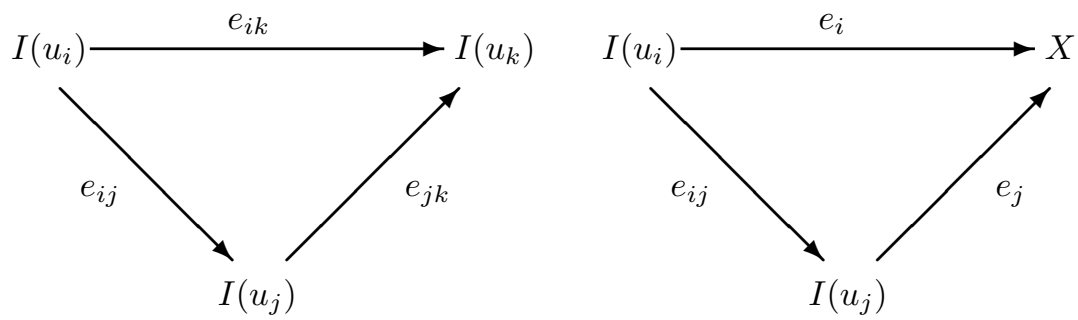

commute. Therefore the system $\left(I\left(u_{i}\right),\left(e_{i j}\right)_{u_{i}<u_{j}}\right)$ is an inductive system of convergence vector spaces, and $\left(r(X), e_{i}\right)_{u_{i}>0}$ is compatible with this system. This leads to the following.

THEOREM 3.4. The convergence vector space $r(X)$ is the convergence inductive limit of the inductive system $\left(I\left(u_{i}\right),\left(e_{i j}\right)_{u_{i}<u_{j}}\right)$.

Proof. Since $X=\bigcup_{u_{i}>0} I\left(u_{i}\right)$, according to [8, Proposition 3.4.2] we need only verify that

$$
\mathcal{F} \in \lambda_{r}(0) \Leftrightarrow\left(\begin{array}{ll}
\exists & u_{i}>0: \\
\exists & \mathcal{G} \text { a filter on } I\left(u_{i}\right) \text { converging to } 0: \\
& e_{i}(\mathcal{G}) \subseteq \mathcal{F} .
\end{array}\right) .
$$

Suppose that a filter $\mathcal{F}$ converges to 0 in $r(X)$ so that, by Corollary 2.2, there exists some $u_{i}>0$ in $X$ so that $\left[\left\{\left[-\frac{1}{n} u_{i}, \frac{1}{n} u_{i}\right]: n \in \mathbb{N}\right\}\right] \subseteq \mathcal{F}$. Note that $\left[-\frac{1}{n} u_{i}, \frac{1}{n} u_{i}\right] \subset$ $I\left(u_{i}\right)$ for all $n \in \mathbb{N}$. Furthermore, the filter $\mathcal{G}=\left[\left\{\left[-\frac{1}{n} u_{i}, \frac{1}{n} u_{i}\right]: n \in \mathbb{N}\right\}\right]$ converges to 0 in $I\left(u_{i}\right)$, and $e_{i}(\mathcal{G}) \subseteq \mathcal{F}$. Therefore (3.5) holds.

Conversely, suppose that (3.5) holds. It follows from the continuity of each of the mappings $e_{i}$ that $\mathcal{F}$ converges to 0 in $r(X)$.

Note that all the spaces $I\left(u_{i}\right)$ are complete if and only if $r(X)$ is complete. In view of Proposition 2.4, it therefore follows that all the $I\left(u_{i}\right)$ are complete if and only if $X$ is relatively uniformly complete. The following is now immediate. 
Corollary 3.5. If $X$ is relatively uniformly complete, then $r(X)$ is the convergence inductive limit of a family of Fréchet spaces.

The final ingredient in the Closed Graph Theorem 3.1 is ultracompleteness. In particular, we must find an ultracomplete vector space convergence structure which is finer than the relatively uniform convergence structure $\lambda_{r}$. This problem can, to a good extent, be reduced to showing that $r(X)$ itself is ultracomplete. In this regard, recall [1] that a set $F \subseteq X$ is solid if $y \in F$ whenever there exists $x \in F$ so that $|y| \leq|x|$. A vector space convergence structure on $X$ is called locally solid [17, Definition 2.1] whenever each filter converging to 0 contains a filter with a basis of solid sets that also converges to 0 . The concept of a local solid convergence structure generalizes the well known notion of a locally solid topology on a vector lattice, see for instance [2].

Proposition 3.6. If $\lambda$ is a locally solid convergence structure on $X$, then $\lambda_{r}$ is finer than $\lambda$.

Proof. Let $\lambda$ be a locally solid convergence structure on $X$. Consider any $\mu>0$ in $X$. We claim that the filter $\mathcal{F}=\left[\left\{\left[-\frac{1}{n} \mu, \frac{1}{n} \mu\right]: n \in \mathbb{N}\right\}\right]$ converges to 0 with respect to $\lambda$. In this regard, we note that the filter $\mathcal{N} \mu$ converges to 0 in $X$ with respect to any vector space convergence structure, thus in particular with respect to $\lambda$, see [8, Proposition 3.2.3]. Since $\lambda$ is locally solid, it follows that there is some filter $\mathcal{G}$ with a basis $\mathcal{B}$ of solid sets so that $\mathcal{G}$ converges to 0 with respect to $\lambda$ and $\mathcal{G} \subseteq \mathcal{N} u$. Thus we have

$$
\begin{aligned}
\forall & B \in \mathcal{B}: \\
\exists & n_{B} \in \mathbb{N}: \\
& \left\{\alpha \mu:|\alpha| \leq \frac{1}{n}\right\} \subseteq B .
\end{aligned}
$$

Since every $B \in \mathcal{B}$ is solid, it follows from (3.6) that $\left[-\frac{1}{n} \mu, \frac{1}{n} \mu\right] \subseteq B$ for all $n \geq n_{B}$. Thus $\mathcal{G} \subseteq \mathcal{F}$ so that $\mathcal{F} \in \lambda(0)$. Therefore $\lambda_{r}$ is finer than $\lambda$.

Proposition 3.7. Let $X$ be relatively uniformly complete. Then $r(X)$ is ultracomplete if and only if $r(X)$ is strongly first countable.

Proof. Let $\mathcal{F}$ converge to 0 in $r(X)$. In fact, it is sufficient to consider a filter of the form $\mathcal{F}=\left[\left\{\left[-\frac{1}{n} \mu, \frac{1}{n} \mu\right]: n \in \mathbb{N}\right\}\right]$ for some $\mu \in X^{+}$. Consider the collection

$$
\left\{W_{n}: n \in \mathbb{N}\right\} \subset \mathcal{F}
$$

where $W_{n}=\left[-\frac{1}{2^{n}} \mu, \frac{1}{2^{n}} \mu\right], n \in \mathbb{N}$. We claim that the filter

$$
\left[\left\{\sum_{k=n}^{\infty} W_{k}: n \in \mathbb{N}\right\}\right]
$$

converges to 0 in $r(X)$. We first show that the elements of this filter are well-defined. In this regard, consider any sequence $\left(x_{n}\right)$ such that $x_{n} \in W_{n}$ for every $n \in \mathbb{N}$. 
Define the sequence $\left(y_{k}\right)$ in $X$ by setting $y_{k}=\sum_{n=1}^{k} x_{n}$ for every $k \in \mathbb{N}$. The filter $\left\langle y_{k}\right\rangle-\left\langle y_{k}\right\rangle$ is based on the collection of sets $\left\{\left\{ \pm \sum_{n=N}^{N+m} x_{n}: m \in \mathbb{N}\right\}: N \in \mathbb{N}\right\}$. Note that, since $x_{n} \in W_{n}$ for all $n \in \mathbb{N}$,

$$
-\sum_{n=N}^{N+m} \frac{1}{2^{n}} \mu \leq \sum_{n=N}^{N+m} x_{n} \leq \sum_{n=N}^{N+m} \frac{1}{2^{n}} \mu .
$$

Therefore

$$
-\frac{1}{2^{N-1}} \mu \leq \sum_{n=N}^{N+m} x_{n} \leq \frac{1}{2^{N-1}} \mu
$$

for all $N \in \mathbb{N}$ so that $\mathcal{G}=\left[\left\{\left[-\frac{1}{2^{N-1}} \mu, \frac{1}{2^{N-1}} \mu\right]: N \in \mathbb{N}\right\}\right] \subseteq\left\langle y_{k}\right\rangle-\left\langle y_{k}\right\rangle$. Consequently $\left\langle y_{k}\right\rangle-\left\langle y_{k}\right\rangle$ converges to 0 in $r(X)$. Hence $\left(y_{k}\right)$ is a Cauchy sequence in $r(X)$. But $r(X)$ is complete by Proposition 2.4 so that $\left(y_{k}\right)$ converges to some $y$ in $r(X)$. Therefore the elements of the filter (3.7) are well defined. Since

$$
\sum_{k=n}^{\infty} W_{k} \subseteq\left[-\sum_{k=n}^{\infty} \frac{1}{2^{k}} \mu, \sum_{k=n}^{\infty} \frac{1}{2^{k}} \mu\right]=\left[-\frac{1}{2^{n-1}} \mu, \frac{1}{2^{n-1}} \mu\right]
$$

for every $n \in \mathbb{N}$, it follows that

$$
\mathcal{F}=\left[\left\{\left[-\frac{1}{2^{n-1}} \mu, \frac{1}{2^{n-1}} \mu\right]: n \in \mathbb{N}\right\}\right] \subseteq\left[\left\{\sum_{k=n}^{\infty} W_{k}: n \in \mathbb{N}\right\}\right] .
$$

But $\mathcal{F}$ converges to 0 in $r(X)$. Therefore the filter $\left[\left\{\sum_{k=n}^{\infty} W_{k}: n \in \mathbb{N}\right\}\right]$ converges to 0 in $r(X)$. It follows from the definition of ultracompleteness [8, Definition 6.1.1] $r(X)$ is ultracomplete if and only if $r(X)$ is strongly first countable.

Proposition 3.8. $r(X)$ is strongly first countable if and only if there is a countable set $B=\left\{u_{n}: n \in \mathbb{N}\right\} \subset X^{+}$so that $X$ is the ideal generated by $B$.

Proof. Assume that $X$ is the ideal generated by a countable set

$$
B=\left\{u_{n}: n \in \mathbb{N}\right\} \subset X^{+} .
$$

Then it is easy to see that the collection of intervals $\left\{\left[-\frac{k}{m} u_{n}, \frac{k}{m} u_{n}\right]: k, m, n \in \mathbb{N}\right\}$ is a local basis for $\lambda_{r}$ at 0 . Hence, by translation invariance, $r(X)$ has a countable local basis at every $x \in X$, and is thus strongly first countable.

Conversely, assume that $r(X)$ is strongly first countable. Let $\mathcal{B}=\left\{B_{n}: n \in \mathbb{N}\right\}$ be a countable local basis for $\lambda_{r}$ at 0 . Without loss of generality, we may assume that every member of $\mathcal{B}$ belongs to at least one filter in $\lambda_{r}(0)$. Consider a filter $\mathcal{F} \in \lambda_{r}(0)$ with basis $\mathcal{B}_{\mathcal{F}}=\left\{B_{n_{k}}: k \in \mathbb{N}\right\} \subseteq \mathcal{B}$. According to Corollary 2.2

$$
\begin{array}{ll}
\exists & v \in X^{+}: \\
\forall & m \in \mathbb{N}: \\
\exists & k_{m} \in \mathbb{N}: \\
& B_{n_{k_{m}}} \subseteq\left[-\frac{1}{m} v, \frac{1}{m} v\right] .
\end{array}
$$


It follows from (3.8) that the filter

$$
\left[\left\{B_{n_{k}} \mid \exists \quad \begin{array}{l}
m \in \mathbb{N}: \\
\quad B_{n_{k}} \subseteq\left[-\frac{1}{m} v, \frac{1}{m} v\right]
\end{array}\right\}\right] \subseteq \mathcal{F}
$$

is well defined and, by Corollary 2.2, converges to 0 in $r(X)$. Thus we may assume that the members of $\mathcal{B}$ are order bounded so that

$$
\begin{aligned}
\forall & n \in \mathbb{N}: \\
\exists & u_{n} \in X^{+}: \\
& B_{n} \subseteq\left[-u_{n}, u_{n}\right] .
\end{aligned}
$$

Consider any $u \in X$. Since $\mathcal{B}$ is a local basis for $\lambda_{r}$ at 0 , it follows from Corollary 2.2 that

$$
\begin{array}{ll}
\exists & \left\{B_{n_{k}}: k \in \mathbb{N}\right\} \subseteq \mathcal{B}: \\
\forall & k \in \mathbb{N}: \\
\exists & m_{k} \in \mathbb{N}: \\
& {\left[-\frac{1}{m_{k}}|u|, \frac{1}{m_{k}}|u|\right] \subseteq B_{n_{k}} .}
\end{array}
$$

It now follows from (3.9) that $|u| \leq m_{1} u_{n_{1}}$. Therefore $X$ is the ideal generated by $B=\left\{u_{n}: n \in \mathbb{N}\right\}$.

Corollary 3.9. Let $X$ be a relative uniformly complete. Then $r(X)$ is ultracomplete if and only if there is a countable set $B=\left\{u_{n}: n \in \mathbb{N}\right\} \subset X^{+}$so that $X$ is the ideal generated by $B$.

Proof. This follows directly from Propositions 3.7 and 3.8 .

Using the results obtained in this section, we proceed to establish a closed graph theorem for operators $T: r(X) \rightarrow r(Y)$, with $X$ and $Y$ Archimedean vector lattices. This in turn, leads to a closed graph theorem for order bounded operators.

Theorem 3.10. Let $X$ and $Y$ be relatively uniformly complete Archimedean vector lattices. If there is a countable set $B=\left\{u_{n}: n \in \mathbb{N}\right\} \subset Y^{+}$so that $Y$ is the ideal generated by $B$, then every closed linear mapping $T: r(X) \rightarrow r(Y)$ is continuous.

Proof. The result follows immediately from Corollaries 3.5 and 3.9 and Theorem 3.1.

Corollary 3.11. Let $X$ and $Y$ be relatively uniformly complete Archimedean vector lattices, and $T: X \rightarrow Y$ a linear mapping with the property that for all $x \in X$ and $y \in Y$,

$\left.\begin{array}{ll}(1) & \left(x_{n}\right) \text { converges relatively uniformly to } x \\ (2) & \left(T x_{n}\right) \text { converges relatively uniformly to } y\end{array}\right) \Rightarrow T x=y$. 
If there is a countable set $B=\left\{u_{n}: n \in \mathbb{N} \subset Y^{+}\right\}$so that $Y$ is the ideal generated by $B$, then $T$ is order bounded.

Proof. Since $r(X)$ and $r(Y)$ are first countable, so is $r(X) \times r(Y)$, see [8, Corollary 1.6.8]. Our assumption (3.10), together with [8, Proposition 1.6.4], therefore imply that $T$ is closed. Theorem 3.10 implies that $T$ is continuous. Since $r(X)$ and $r(Y)$ are the Mackey modifications of $o(X)$ and $o(Y)$, respectively, [8, Proposition 3.7.17] implies that a linear mapping from $r(X)$ into $r(Y)$ is continuous if and only if it maps bounded sets in $o(X)$ to bounded sets in $o(Y)$. It therefore follows from Proposition 2.1 that $T$ is order bounded.

4. Conclusion. As mentioned, the topological concepts such as convergence of sequences and continuity of linear mappings that are at the heart of analysis on vector lattices can, in general, not be described in terms of the usual HausdorffKuratowski-Bourbaki topology. However, we have shown in this paper, and in $[16,17]$, that convergence vector spaces provide an appropriate context for the study of such topological notions on vector lattices. Thus the methods of the theory of convergence vector spaces may be applied to vector lattice theory. As an illustration of the utility of these methods, we proved a closed graph theorem for order bounded operators on a class of relatively uniformly complete Archimedean vector lattices. One may note that such a result has by now not been obtained by any other methods.

\section{REFERENCES}

1. C.D. Aliprantis And K.C. Border, Infite dimensional analysis. A hitchhiker's guide, Springer, Berlin, 2006.

2. C.D. Aliprantis And O. Burkinshaw, Locally solid Riesz spaces and applications to economics, 2nd ed., American Mathematical Society, Providence, Rhode Island, 2003.

3. R. Anguelov ANd J.H. VAn Der Walt, Order convergence structure on C (X), Quaestiones Mathematicae 28 (2005), 425-457.

4. S. Banach, Théorie des opérations linéaires, Mathematisches Seminar der Univ. Warschau, Warsaw, 1932.

5. R. Beattie, Continuous convergence and the closed graph theorem, Mathematische Nachrichten 99 (1980), 87-94.

6. of the International Conference on Categorical Topology, Toledo, Ohio, (1983), 29-45, Helderman-Verlag, Berlin, 1984.

7. Continuous convergence and functional analysis, Topology and its Applications 70 (1996), 101-111.

8. R. Beattie And H.P. Butzmann, Convergence structures and applications to functional analysis, Kluwer Academic Publishers, Dordrecht, 2002. 
9. M. DE WILDE, Closed graph theorems and webbed spaces, Pitman, Boston, Massachusetts, 1978.

10. W.A.J. Luxemburg And A.C. ZaAnen, Riesz spaces I, North Holland, Amsterdam, 1971.

11. E.T. Ordman, Convergence almost everywhere is not topological, American Mathematical Montly 73 (1966), 182-183.

12. V. PтÁk, Completeness and the open mapping theorem, Bulletin de la Société Mathématique de France 86 (1958), 41-74.

13. D.A. RĂ̌KOV, A two-sided closed graph theorem for topological linear spaces, Sibirskii Matematičeskii Žurnal 7 (1966), 353-372.

14. H.H. SchaEFER, Banach lattices and positive operators, Springer-Verlag, Berlin/Heidelberg/New York, 1974.

15. W. SŁOWIKowski, On continuity of inverse operators, Bulletin of the American Mathematical Society 67 (1961), 467-470.

16. J.H. VAN DER WALT, The order convergence structure, Indagationes Mathematicae (N.S.) 21 (2011), 138-155.

17. Applications of convergence structures to vector lattice theory, Topolgy Proceedings 41 (2013), 311-331. 